\title{
ENDEMIC BEHAVIOUR OF SIS EPIDEMICS WITH GENERAL INFECTIOUS PERIOD DISTRIBUTIONS
}

\author{
PETER NEAL,${ }^{*}$ Lancaster University
}

\begin{abstract}
We study the endemic behaviour of a homogeneously mixing SIS epidemic in a population of size $N$ with a general infectious period, $Q$, by introducing a novel subcritical branching process with immigration approximation. This provides a simple but useful approximation of the quasistationary distribution of the SIS epidemic for finite $N$ and the asymptotic Gaussian limit for the endemic equilibrium as $N \rightarrow \infty$. A surprising observation is that the quasistationary distribution of the SIS epidemic model depends on $Q$ only through $\mathbb{E}[Q]$.
\end{abstract}

Keywords: SIS epidemic; branching process with immigration; Gaussian process; quasistationary distribution

2010 Mathematics Subject Classification: Primary 92D30

Secondary 60G10

\section{Introduction}

The SIS epidemic model is the simplest epidemic model which exhibits endemic behaviour, and has consequently received considerable interest; see, for example, Weiss and Dishon (1971), Kryscio and Lefèvre (1989), Andersson and Djehiche (1998), Clancy and Pollett (2003), and Clancy and Mendy (2011). In its simplest form there is assumed to be a homogeneously mixing closed population of $N$ individuals, partitioned into two groups: susceptibles and infectives. Whilst infectious, an infective makes infectious contacts at the points of a homogeneous Poisson point process with rate $\beta$ with the individual contacted chosen uniformly at random from the entire population. Infectious contacts with susceptibles result in the recipient becoming infected and instantaneously infectious, whilst infectious contacts with infectives have no effect on the recipient. Infectives have a constant recovery rate, $\gamma$, at which they recover from the disease and return to the susceptible state. This implies that the infectious period of infectives is exponentially distributed with mean $\gamma^{-1}$ and that the epidemic process is Markovian.

A number of key results are known for the SIS epidemic model, both for finite $N$ and asymptotic as $N \rightarrow \infty$. For finite $N$, the quasistationary distribution of the epidemic process (see Kryscio and Lefèvre (1989), Nåsell (1996), (1999a), Clancy and Pollett (2003), Clancy and Mendy (2011), and Clancy (2012)) and the time to extinction from the endemic equilibrium (see Andersson and Djehiche (1998) and Nåsell (1999a)) have been investigated since, almost surely, the SIS epidemic eventually goes extinct. Asymptotic results as $N \rightarrow \infty$ are a branching process approximation for the initial stages of the epidemic and a Gaussian process limit, using Theorem 3.5 of Kurtz (1971), for the fluctuations of the stochastic process about the deterministic limit. The branching process approximation can be obtained using a similar approach to that given in Ball and Donnelly (1995) for the SIR epidemic; see, for example,

Received 20 December 2012; revision received 21 March 2013.

* Postal address: Department of Mathematics and Statistics, Fylde College, Lancaster University, Lancaster LA1 4YF, UK. Email address: p.neal@lancaster.ac.uk 
Ball (1999). The Gaussian process limit leads to an Ornstein-Uhlenbeck limit about the endemic equilibrium should one exist; see Kryscio and Lefèvre (1989). The branching process approximation mirrors that for the SIR epidemic model, whereas the other results are exclusively for the SIS epidemic model. The Markov SIS epidemic model has been studied amongst heterogeneous populations, the household epidemic model (see Ball (1999), Ghoshal et al. (2004), Neal (2006), Arrigoni and Pugliese (2007), and Britton and Neal (2010)) and the great circle epidemic model (see Neal (2008)). For these epidemic models, a branching process approximation for the initial stages of the epidemic (see Ball (1999) and Neal (2008)), stability of the disease free equilibrium (see Ball (1999) and Ghoshal et al. (2004)), the endemic equilibrium (see Ball (1999), Ghoshal et al. (2004), and Neal (2006), (2008)), an OrnsteinUhlenbeck limit about the endemic equilibrium (see Ball (1999), Neal (2006), and Britton and Neal (2010)) and the time to extinction (see Britton and Neal (2010)) have been investigated.

In this paper we consider generalising the SIS epidemic model in a different direction by assuming that the population is homogeneously mixing but that the infectious periods are independent and identically distributed (i.i.d.) according to an arbitrary, but specified, nonnegative probability distribution $Q$. As noted above, it is trivial to adapt the branching process approximation for an SIR epidemic model with general infectious period (see Ball and Donnelly (1995)) to an SIS epidemic model. Therefore, we focus on the endemic behaviour of the SIS epidemic model, the (asymptotic) proportion infectious in endemic equilibrium, the quasistationary distribution of the epidemic and an asymptotic Gaussian limit about the endemic equilibrium.

For the SIR epidemic model, the basic reproduction number $R_{0}$ and the asymptotic proportion infected by a major epidemic outbreak are known to depend on $Q$, the infectious period distribution, only through $\mathbb{E}[Q]$. In contrast, the probability that a major epidemic occurs and the asymptotic variance of the proportion infected in a major epidemic depend on the distributional form of $Q$ and not just $\mathbb{E}[Q]$. For the endemic level of the SIS epidemic model, we show that not only the proportion infected at the endemic level but also the approximating quasistationary distribution, and the corresponding endemic equilibrium distribution depend on $Q$ only through $\mathbb{E}[Q]$. A Gaussian limit for the evolution of the epidemic process about its endemic equilibrium is obtained and this does depend on the distributional form of $Q$. Without loss of generality, throughout the paper, we set $\mathbb{E}[Q]=1$, as changing $\mathbb{E}[Q]$ only changes the timescale of the epidemic if $\beta$ is adjusted accordingly.

The paper is structured as follows. In Section 2 we consider the equilibrium behaviour of a subcritical branching process with immigration, in particular, the stationary distribution for finite immigration rate and the asymptotic behaviour as the immigration rate $\alpha \rightarrow \infty$. The subcritical branching process with immigration provides a novel and useful approximation for the SIS epidemic model about the endemic equilibrium. In Section 3 we exploit this approximation to study the endemic equilibrium of the SIS epidemic model with $1<R_{0}<2$, and obtain a simple approximation of the quasistationary distribution of the epidemic model. The approximating quasistationary distribution obtained is a negative binomial distribution which is similar to that obtained in Clancy and Mendy (2011) by totally different methods (cumulant equations). Our approximation is valid for all choices of $Q$, whereas the construction of Clancy and Mendy (2011) explicitly relies upon $Q$ being exponentially distributed. The case $1<R_{0}<2$ corresponds to an endemic level $0<\rho=\left(R_{0}-1\right) / R_{0}<\frac{1}{2}$, which is the most realistic scenario for an endemic SIS disease. Finally, in Section 4 we summarise the results obtained and briefly discuss how the distribution of $Q$ affects the mean time to extinction of the epidemic. 


\section{Subcritical branching process with immigration}

For $N \geq 1$, we consider a sequence of branching processes $\left\{\mathcal{B}_{N}\right\}$, indexed by $N$, which are defined as follows. Individuals immigrate into the population at the points of a homogeneous Poisson process at rate $N \alpha$. Each individual on entering the population has an i.i.d. life history according to $(Q, \eta)$. Let $Q$ be an arbitrary, but specified, nonnegative distribution denoting the length of an individual's lifetime. Without loss of generality, we set $\mathbb{E}[Q]=1$. Let $\eta$ be a homogeneous Poisson point process with rate $\delta$ of times, relative to the individual's entry into the population either by immigration or birth, at which the individual gives birth. Thus, an individual, $i$ say, with life history $\left(Q_{i}, \eta_{i}\right)$, who enters the population at time, $t$ say, gives birth to new individuals at the points of $t+\eta_{i}$ before dying at $t+Q_{i}$. To ensure that the branching process is subcritical, we require that $\delta<1$. We assume that the population configuration at time 0 is from the stationary distribution of $\mathscr{B}_{N}$, which can be obtained by running the branching process from $t=-\infty$.

Let $B_{N}(t)$ denote the total number of individuals alive in the branching process $\mathscr{B}_{N}$ at time $t$. It is straightforward to show, using stationarity, that $\mathbb{E}\left[B_{N}(t)\right]=\mathbb{E}\left[B_{N}(0)\right]=\alpha N /(1-$ $\delta)$. We begin by obtaining the probability generating function of $B_{N}(t)$ in Lemma 2.1, and, consequently, show that $B_{N}(t)$ follows a negative binomial distribution. This is followed in Corollary 2.1 by a central limit theorem for $Z_{N}(t)=\left(B_{N}(t)-\mathbb{E}\left[B_{N}(t)\right]\right) / \sqrt{N}$ as $N \rightarrow \infty$, which is extended in Theorem 2.1 to a Gaussian process limit for $Z_{N}(\cdot)$.

For $u \geq 0$, let $X(u)$ denote the total number of individuals alive in a branching process $\mathcal{X}$ where there is one initial ancestor who enters the population at time 0 and individual histories are i.i.d. according to $(Q, \eta)$. Thus, $\{X(u)\}$ represents the family history of an immigrant in $\mathscr{B}_{N}$, relative to the immigrant's arrival time. For $0 \leq s \leq 1$ and $u \geq 0$, let $\phi(s, u)=\mathbb{E}\left[s^{X(u)}\right]$. In the sequel $\mathcal{X}$, and, hence, $X(u)$ and $\phi(s, u)$ play key roles. It is well known that, if $Q \sim \operatorname{Exp}(1)$, using the Kolmogorov backward equations,

$$
\phi(s, u)=\frac{(1-s)-(1-\delta s) \exp ((1-\delta) u)}{\delta(1-s)-(1-\delta s) \exp ((1-\delta) u)} .
$$

It is also clear that $X(u)$ and, hence, $\phi(s, u)$ depends upon $Q$. For example, $\mathbb{P}(X(u) \geq 1)=1$ for $u<1$ if $Q \equiv 1$, which is certainly not the case for $Q \sim \operatorname{Exp}(1)$. However, an important quantity is

$$
\begin{aligned}
\int_{0}^{\infty}\{\phi(s, u)-1\} \mathrm{d} u & =\int_{0}^{\infty}\left\{\sum_{k=0}^{\infty} s^{k} \mathbb{P}(X(u)=k)-\sum_{k=0}^{\infty} \mathbb{P}(X(u)=k)\right\} \mathrm{d} u \\
& =\sum_{k=0}^{\infty}\left(s^{k}-1\right) \int_{0}^{\infty} \mathbb{P}(X(u)=k) \mathrm{d} u \\
& =\sum_{k=1}^{\infty}\left(s^{k}-1\right) \int_{0}^{\infty} \mathbb{P}(X(u)=k) \mathrm{d} u
\end{aligned}
$$

which is governed by $\int_{0}^{\infty} \mathbb{P}(X(u)=k) \mathrm{d} u$, the mean amount of time the branching process $\mathcal{X}$ spends with $k$ individuals alive. Somewhat surprisingly, it appears that $\int_{0}^{\infty} \mathbb{P}(X(u)=k) \mathrm{d} u$ depends on $Q$ only through $\mathbb{E}[Q]$.

Conjecture 2.1. Given that $\mathbb{E}[Q]=1$. For all $k=1,2, \ldots$,

$$
\int_{0}^{\infty} \mathbb{P}(X(u)=k) \mathrm{d} u=\frac{\delta^{k-1}}{k} .
$$


Proof. The general statement of the conjecture has alluded full proof. We present a proof of (2.2) in the case $Q \sim \operatorname{Exp}(1)$ and outline results which support the conjecture.

Since, for $0 \leq s \leq 1$,

$$
\sum_{k=1}^{\infty}\left(s^{k}-1\right) \frac{\delta^{k-1}}{k}=\frac{1}{\delta} \log \left(\frac{1-\delta}{1-s \delta}\right)
$$

the conjecture holds if

$$
\int_{0}^{\infty}\{\phi(s, u)-1\} \mathrm{d} u=\frac{1}{\delta} \log \left(\frac{1-\delta}{1-s \delta}\right) .
$$

Using (2.3) it is trivial to see that the conjecture holds for $(Q, \delta=0)$ for any $Q$, where $\phi(s, u)=1+(s-1) \mathbb{P}(Q>u)$.

For $Q \sim \operatorname{Exp}(1)$, using (2.1),

$$
\int_{0}^{\infty}\{\phi(s, u)-1\} \mathrm{d} u=\int_{0}^{\infty} \frac{(1-\delta)(1-s)}{\delta(1-s)-(1-s \delta) \exp ((1-\delta) u)} \mathrm{d} u
$$

By a change of variable with $y=\exp ((1-\delta) u)$ and using partial fractions, we have $\mathrm{d} y / y=$ $(1-\delta) \mathrm{d} u$ and

$$
\begin{aligned}
\int_{0}^{\infty}\{\phi(s, u)-1\} \mathrm{d} u & =\int_{1}^{\infty} \frac{(1-s)}{y\{\delta(1-s)-(1-s \delta) y\}} \mathrm{d} y \\
& =(1-s) \int_{1}^{\infty}\left\{\frac{1}{\delta(1-s) y}+\frac{1-s \delta}{\delta(1-s)\{\delta(1-s)-(1-s \delta) y\}}\right\} \mathrm{d} y \\
& =\frac{1}{\delta} \int_{1}^{\infty}\left\{\frac{1}{y}+\frac{1-s \delta}{\delta(1-s)-(1-s \delta) y}\right\} \mathrm{d} y \\
& =\frac{1}{\delta}[\log (y)-\log |\delta(1-s)-(1-s \delta) y|]_{1}^{\infty} \\
& =\frac{1}{\delta}\left[\log \left(\frac{y}{|\delta(1-s)-(1-s \delta) y|}\right)\right]_{1}^{\infty} \\
& =\frac{1}{\delta}\left\{\log \left(\frac{1}{1-s \delta}\right)-\log \left(\frac{1}{1-\delta}\right)\right\} \\
& =\frac{1}{\delta} \log \left(\frac{1-\delta}{1-s \delta}\right)
\end{aligned}
$$

as required by (2.3).

For $Q \sim \operatorname{Exp}\left(\gamma_{1}\right)+\operatorname{Exp}\left(\gamma_{2}\right)$ with $\gamma_{1}^{-1}+\gamma_{2}^{-1}=1$ and $\delta \geq 0$, we can model the individuals as having a two-stage lifetime: one stage of length $\operatorname{Exp}\left(\gamma_{1}\right)$ followed by another stage of length $\operatorname{Exp}\left(\gamma_{2}\right)$. Then $\phi(s, u)=\varphi_{1}((s, s), u)$, where $\varphi_{k}(z, u)(k=1,2)$ denotes the probability generating function for the total number of individuals in each life stage at time $u$ given that there is an initial ancestor in life stage $k$ at time 0 . Note that $\varphi_{1}(z, u)$ and $\varphi_{2}(z, u)$ solve the backward Kolmogorov equations

$$
\begin{aligned}
& \frac{\partial \varphi_{1}(z, t)}{\partial t}=\delta \varphi_{1}(z, t)^{2}+\gamma_{1} \varphi_{2}(z, t)-\left(\delta+\gamma_{1}\right) \varphi_{1}(z, t), \\
& \frac{\partial \varphi_{2}(z, t)}{\partial t}=\delta \varphi_{1}(z, t) \varphi_{2}(z, t)+\gamma_{2}-\left(\delta+\gamma_{2}\right) \varphi_{2}(z, t)
\end{aligned}
$$


subject to the initial conditions $\varphi_{k}(z, 0)=z_{k}$. Using MAPLE ${ }^{\circledR} 16$, it is possible to get an (long and unwieldy) analytic expression for $\phi(s, u)$ with numerical integration used to evaluate (2.3) for a wide range of choices of $\delta, \gamma_{1}, \gamma_{2}$ and $s$ to verify that (2.3) holds.

It is difficult to prove analytically the conjecture for general $Q$, although it is possible to show that, for any $Q$ with $\mathbb{E}[Q]=1$ and $\delta$ small, but positive, $\int_{0}^{\infty} \mathbb{P}(X(u)=2)=\delta / 2+o(\delta)$. Thus, the result was checked using Monte Carlo estimation of $\int_{0}^{\infty} \mathbb{P}(X(u)=k) \mathrm{d} u$ using a range of choices of $Q$ and $\delta$ with 10000 simulations used for each combination. In all cases the results were consistent with (2.2) with small Monte Carlo error.

We proceed under the assumption that Conjecture 2.1 holds.

Lemma 2.1. For any $Q$ such that $\mathbb{E}[Q]=1$, the unique stationary distribution $B_{N}^{*}$ for the branching process $\mathscr{B}_{N}$ satisfies, for all $0 \leq s \leq 1$,

$$
\mathbb{E}\left[s^{B_{N}^{*}}\right]=\exp \left(\alpha N \int_{0}^{\infty}\{\phi(s, u)-1\} \mathrm{d} u\right)=\left(\frac{1-\delta}{1-s \delta}\right)^{\alpha N / \delta} .
$$

Proof. Since $\mathcal{B}_{N}$ starts in stationarity, $B_{N}(0) \stackrel{\mathrm{D}}{=} B_{N}^{*}$ and we consider $\mathbb{E}\left[s^{B_{N}(0)}\right]$.

For $i=1,2, \ldots$, let $\mathcal{C}_{i}=[-i,-(i-1))$. Then the total number of individuals immigrating into the population in $\mathcal{C}_{i}$ is $C_{i} \sim \operatorname{Po}(\alpha N)$ and the immigration times are uniformly distributed over $\mathcal{C}_{i}$. Let $-U_{i, j} \sim U[-i,-(i-1))\left(j=1,2, \ldots, C_{i}\right)$ denote the immigration of the $j$ th individual in $\mathcal{C}_{i}$. Note that the labelling of the immigrants in $\mathcal{C}_{i}$ does not refer to the time order of the immigrations, instead using a construction which gives i.i.d. immigration times for the $C_{i}$ individuals. Let $X^{i, j}(u)$ denote the total number of individuals in the branching process originating from the $j$ th immigrant in $\mathcal{C}_{i}$ at $u$ units of time after immigration. Then $B_{N}(0)=\sum_{i=1}^{\infty} \sum_{j=1}^{C_{i}} X^{i, j}\left(U_{i, j}\right)$, where, for fixed $i$, the $X^{i, j}\left(U_{i, j}\right)$ are i.i.d. with

$$
\mathbb{E}\left[s^{X^{i, j}\left(U_{i, j}\right)}\right]=\int_{-i}^{-(i-1)} \phi(s, u) \mathrm{d} u .
$$

Since the $C_{i}$ are i.i.d., we have

$$
\begin{aligned}
\mathbb{E}\left[s^{B_{N}(0)}\right] & =\mathbb{E}\left[s^{\sum_{i=1}^{\infty} \sum_{j=1}^{C_{i}} X^{i, j}\left(U_{i, j}\right)}\right] \\
& =\prod_{i=1}^{\infty} \mathbb{E}\left[\mathbb { E } \left[s^{\left.\left.X^{i, 1}\left(U_{i, 1}\right)\right]^{C_{i}}\right]}\right.\right. \\
& =\prod_{i=1}^{\infty} \mathbb{E}\left[\left\{\int_{-i}^{-(i-1)} \phi(s,-u) \mathrm{d} u\right\}^{C_{i}}\right] \\
& =\prod_{i=1}^{\infty} \exp \left(\alpha N\left\{\int_{-i}^{-(i-1)} \phi(s,-u) \mathrm{d} u-1\right\}\right) \\
& =\exp \left(\alpha N \sum_{i=1}^{\infty}\left\{\int_{-i}^{-(i-1)} \phi(s,-u) \mathrm{d} u-1\right\}\right) \\
& =\exp \left(\alpha N \int_{0}^{\infty}\{\phi(s, u)-1\} \mathrm{d} u\right) .
\end{aligned}
$$

satisfying (2.4). 
Then, for $r=0,1, \ldots, B_{N}^{*}$ has probability mass function

$$
\mathbb{P}\left(B_{N}^{*}=r\right)=\frac{\Gamma(\alpha N / \delta+r)}{\Gamma(\alpha N / \delta) r !} \delta^{\alpha N / \delta}(1-\delta)^{r},
$$

which, for $\alpha N / \delta \in \mathbb{N}$, gives $B_{N}^{*} \sim \operatorname{NBin}(\alpha N / \delta, 1-\delta)$. Throughout we use the term negative binomial distribution to refer to the generalised distribution given by (2.5), and note that $\mathbb{E}\left[B_{N}^{*}\right]=N \alpha /(1-\delta)$ and $\operatorname{var}\left(B_{N}^{*}\right)=N \alpha /(1-\delta)^{2}$.

An immediate corollary of Lemma 2.1 concerns the asymptotic distribution of

$$
Z_{N}^{*}=\frac{B_{N}^{*}-\mathbb{E}\left[B_{N}^{*}\right]}{\sqrt{N}}=\frac{B_{N}^{*}-N \alpha /(1-\delta)}{\sqrt{N}} .
$$

Corollary 2.1. We have

$$
Z_{N}^{*} \stackrel{\mathrm{D}}{\rightarrow} Z^{*} \sim N\left(0, \alpha \int_{0}^{\infty} \mathbb{E}\left[X(u)^{2}\right] \mathrm{d} u\right)=N\left(0, \frac{\alpha}{(1-\delta)^{2}}\right) \quad \text { as } N \rightarrow \infty .
$$

Thus far we have focused upon $B_{N}^{*}$ and $Z_{N}^{*}$ which are independent of the distributional form of $Q$. We now turn to $Z_{N}(\cdot)$ and show that, as $N \rightarrow \infty, Z_{N}(\cdot)$ converges weakly to a Gaussian process $Z(\cdot)$, where the covariance function of $Z(\cdot)$ does depend upon the distributional form of $Q$. To prove this, we proceed as in Scalia-Tomba (1990), by defining, for $N \geq 1, Z_{N}$ on $D[0, \infty)$ endowed with the Skorokhod topology (see Billingsley (1968) and Lindvall (1973)). Note that with a suitable choice of metric, $D[0, \infty)$ becomes a complete, separable metric space. We show that the finite-dimensional distributions of $Z_{N}(\cdot)$ converge weakly to those of $Z(\cdot)$ (Lemma 2.3), and then, for any $T>0, Z_{N} \Rightarrow Z$ on $D[0, T]$ as $N \rightarrow \infty$ (Theorem 2.1).

Before we state and prove Lemma 2.3, a central limit theorem for the finite-dimensional distributions of $Z_{N}(\cdot)$, we give a simple but useful result.

Lemma 2.2. For any $\lambda>0$ and $n \geq 1$, let $X_{n} \sim \operatorname{Po}(\lambda n)$ and let $Y_{1}, Y_{2}, \ldots$ be i.i.d. according to $Y$ with $\mathbb{E}\left[Y^{2}\right]<\infty$. Then, as $n \rightarrow \infty$,

$$
Z_{n}=\frac{1}{\sqrt{n}}\left(\sum_{i=1}^{X_{n}} Y_{i}-\mathbb{E}\left[X_{n}\right] \mathbb{E}[Y]\right) \stackrel{\mathrm{D}}{\rightarrow} Z \sim N\left(0, \lambda \mathbb{E}\left[Y^{2}\right]\right) \text { as } n \rightarrow \infty .
$$

Proof. The lemma is straightforward to prove using convergence of moment generating functions; hence, the details are omitted.

Lemma 2.3. For all $m \in \mathbb{N}$ and $\boldsymbol{t} \in \mathbb{R}^{m}$ with $0\left(=t_{0}\right) \leq t_{1}<t_{2}<\cdots<t_{m}$, as $N \rightarrow \infty$,

$$
\boldsymbol{Z}_{N}(\boldsymbol{t})=\left(Z_{N}\left(t_{1}\right), Z_{N}\left(t_{2}\right), \ldots, Z_{N}\left(t_{m}\right)\right)
$$

converges in distribution to a multivariate normal distribution with mean $\mathbf{0}$ and covariance matrix $\Sigma(\boldsymbol{t})$, with the $(k, l)$ th element of $\Sigma(\boldsymbol{t})$ equal to

$$
\alpha \int_{-\infty}^{t_{m}} v\left(t_{k}-u, t_{l}-u\right) \mathrm{d} u
$$

where, for $s, t \in \mathbb{R}, v(s, t)=\mathbb{E}[X(s) X(t)]$ with $X(t)=0$ for $t<0$. 
Proof. Fix $m \in \mathbb{N}$ and $\boldsymbol{t} \in \mathbb{R}^{m}$. Let $\boldsymbol{\phi}=\left(\phi_{1}, \phi_{2}, \ldots, \phi_{m}\right) \in \mathbb{R}^{m}$ and $V_{N}(\boldsymbol{t}, \boldsymbol{\phi})=$ $\sum_{i=1}^{m} \phi_{i} Z_{N}\left(t_{i}\right)$. For $i=1,2, \ldots, m$, let $\hat{\mathcal{C}}_{i}=\left(t_{i-1}, t_{i}\right]$. Then the total number of individuals immigrating into the population in the interval $\hat{\mathcal{C}}_{i}$ is $\hat{C}_{i}^{N} \sim \operatorname{Po}\left(\alpha N\left(t_{i}-t_{i-1}\right)\right)$ and the immigration times are uniformly distributed over $\hat{\mathcal{C}}_{i}$. Let $\hat{X}^{i, j}(u)$ denote the total number of individuals in the branching process originating from the $j$ th immigrant in $\hat{\mathcal{C}}_{i}$ at $u$ units of time after immigration. Note that, for $u<0, \hat{X}^{i, j}(u)=0$.

For $i=1,2, \ldots, m$ and $j=1,2, \ldots, \hat{C}_{i}^{N}$, let $\hat{U}^{i, j} \sim U\left(t_{i-1}, t_{i}\right)$. Then

$$
\begin{aligned}
V_{N}(\boldsymbol{t}, \boldsymbol{\phi})= & \sum_{i=1}^{m} \phi_{i} Z_{N}\left(t_{i}\right) \\
= & \frac{1}{\sqrt{N}} \sum_{k=1}^{m} \phi_{k}\left\{\sum_{i=1}^{\infty}\left(\sum_{j=1}^{C_{i}^{N}} X^{i, j}\left(t_{k}-U^{i, j}\right)-\mathbb{E}\left[C_{i}^{N}\right] \mathbb{E}\left[X^{i, 1}\left(t_{k}-U^{i, 1}\right)\right]\right)\right. \\
& \left.+\sum_{i=1}^{m}\left(\sum_{j=1}^{\hat{C}_{i}^{N}} \hat{X}^{i, j}\left(t_{k}-\hat{U}^{i, j}\right)-\mathbb{E}\left[\hat{C}_{i}^{N}\right] \mathbb{E}\left[\hat{X}^{i, 1}\left(t_{k}-\hat{U}^{i, 1}\right)\right]\right)\right\} \\
= & \sum_{i=1}^{\infty} \frac{1}{\sqrt{N}}\left\{\sum_{j=1}^{C_{i}^{N}} \sum_{k=1}^{m} \phi_{k} X^{i, j}\left(t_{k}-U^{i, j}\right)-\mathbb{E}\left[C_{i}^{N}\right] \sum_{k=1}^{m} \phi_{k} \mathbb{E}\left[X^{i, 1}\left(t_{k}-U^{i, 1}\right)\right]\right\} \\
& +\sum_{i=1}^{m} \frac{1}{\sqrt{N}}\left\{\sum_{j=1}^{m} \sum_{k=1}^{m} \phi_{k}\left(\hat{X}^{i, j}\left(t_{k}-\hat{U}^{i, j}\right)-\mathbb{E}\left[\hat{C}_{i}^{N}\right] \sum_{k=1}^{m} \phi_{k} \mathbb{E}\left[\hat{X}^{i, 1}\left(t_{k}-\hat{U}^{i, 1}\right)\right]\right\}\right.
\end{aligned}
$$

By Lemma 2.2, for each $i$,

$$
\begin{aligned}
\frac{1}{\sqrt{N}} & \left\{\sum_{j=1}^{C_{i}^{N}} \sum_{k=1}^{m} \phi_{k} X^{i, j}\left(t_{k}-U^{i, j}\right)-\mathbb{E}\left[C_{i}^{N}\right] \sum_{k=1}^{m} \phi_{k} \mathbb{E}\left[X^{i, 1}\left(t_{k}-U^{i, 1}\right)\right]\right\} \\
& \stackrel{\mathrm{D}}{\rightarrow} N\left(0, \alpha \mathbb{E}\left[\left\{\sum_{k=1}^{m} \phi_{k} X^{i, 1}\left(t_{k}-U^{i, 1}\right)\right\}^{2}\right]\right)
\end{aligned}
$$

and

$$
\begin{aligned}
\frac{1}{\sqrt{N}} & \left\{\sum_{j=1}^{\hat{C}_{i}^{N}} \sum_{k=1}^{m} \phi_{k}\left(\hat{X}^{i, j}\left(t_{k}-\hat{U}^{i, j}\right)-\mathbb{E}\left[\hat{C}_{i}^{N}\right] \sum_{k=1}^{m} \phi_{k} \mathbb{E}\left[\hat{X}^{i, 1}\left(t_{k}-\hat{U}^{i, 1}\right)\right]\right\}\right. \\
& \stackrel{\mathrm{D}}{\rightarrow} N\left(0, \alpha\left(t_{i}-t_{i-1}\right) \mathbb{E}\left[\left\{\sum_{k=1}^{m} \phi_{k} \hat{X}^{i, 1}\left(t_{k}-\hat{U}^{i, 1}\right)\right\}^{2}\right]\right)
\end{aligned}
$$

as $N \rightarrow \infty$. Now

$$
\begin{aligned}
\alpha \mathbb{E}\left[\left\{\sum_{k=1}^{m} \phi_{k} X^{i, 1}\left(t_{k}-U^{i, 1}\right)\right\}^{2}\right] & =\alpha \sum_{k=1}^{m} \sum_{l=1}^{m} \phi_{k} \phi_{l} \mathbb{E}\left[X^{i, 1}\left(t_{k}-U^{i, 1}\right) X^{i, 1}\left(t_{l}-U^{i, 1}\right)\right] \\
& =\sum_{k=1}^{m} \sum_{l=1}^{m} \phi_{k} \phi_{l} \alpha \int_{-i}^{-(i-1)} v\left(t_{k}-u, t_{l}-u\right) \mathrm{d} u
\end{aligned}
$$


and

$$
\begin{aligned}
\alpha\left(t_{i}-\right. & \left.t_{i-1}\right) \mathbb{E}\left[\left\{\sum_{k=1}^{m} \phi_{k} \hat{X}^{i, 1}\left(t_{k}-\hat{U}^{i, 1}\right)\right\}^{2}\right] \\
= & \alpha\left(t_{i}-t_{i-1}\right) \sum_{k=1}^{m} \sum_{l=1}^{m} \phi_{k} \phi_{l} \int_{t_{i-1}}^{t_{i}} \frac{1}{t_{i}-t_{i-1}} v\left(t_{k}-u, t_{l}-u\right) \mathrm{d} u \\
& =\sum_{k=1}^{m} \sum_{l=1}^{m} \phi_{k} \phi_{l} \alpha \int_{t_{i-1}}^{t_{i}} v\left(t_{k}-u, t_{l}-u\right) \mathrm{d} u .
\end{aligned}
$$

Therefore, combining (2.7)-(2.11) with the fact that the evolution of each branching process is independent, we have

$$
V_{N}(\boldsymbol{t} ; \boldsymbol{\phi}) \stackrel{\mathrm{D}}{\rightarrow} N\left(0, \sum_{k=1}^{m} \sum_{l=1}^{m} \phi_{k} \phi_{l} \alpha \int_{-\infty}^{t_{m}} v\left(t_{k}-u, t_{l}-u\right) \mathrm{d} u\right) \quad \text { as } N \rightarrow \infty .
$$

The lemma follows by applying the Cramér-Wold device (see Billingsley (1968, pp. 48-49)) to (2.12). This completes the proof.

We exploit the fact that $Z$ is a stationary Gaussian process to prove, firstly, that $Z$ has almost surely continuous sample paths, and secondly, that $\left\{Z_{N}\right\}$ satisfies Condition (A) of Aldous (1978). Then $Z_{N} \Rightarrow Z$ as $N \rightarrow \infty$ follows from Corollary 1 of Aldous (1978).

For $t \geq 0$, let

$$
\begin{aligned}
H(t) & =\alpha \int_{-\infty}^{t}\{v(t-u, t-u)-2 v(t-u,-u)+v(-u,-u)\} \mathrm{d} u \\
& =2 \alpha \int_{-\infty}^{t}\{v(-u,-u)-v(t-u,-u)\} \mathrm{d} u \\
& =2 \alpha \int_{-\infty}^{t} \mathbb{E}[X(-u)(X(-u)-X(t-u))] \mathrm{d} u .
\end{aligned}
$$

Then, for all $0 \leq s \leq t, \operatorname{cov}(Z(s), Z(t))=\operatorname{cov}(Z(0), Z(t-s))=H(t-s)$. By Corollary 25.6 of Rogers and Williams (1994, Chapter I), $Z$ has almost surely continuous sample paths if there exist $\theta>0$ and $C<\infty$ such that, for all $t \geq 0$,

$$
H(t) \leq C t^{\theta}
$$

It suffices to prove (2.13) for $0 \leq t \leq 1$ as the result can then easily be extended for all $t \geq 0$. We prove (2.13) in Lemma 2.4 below for $\theta=1$ under the assumption that $Q$ satisfies $\epsilon_{Q}=\sup _{\{x \geq 0\}} f_{Q}(x)<\infty$. This assumption covers most choices of $Q$ made in the epidemic literature including the gamma distribution with shape parameter greater than or equal to 1 . We discuss relaxing the assumption after Lemma 2.4.

Lemma 2.4. For all $0 \leq t \leq 1$ and $\epsilon_{Q}=\sup _{\{x \geq 0\}} f_{Q}(x)<\infty$,

$$
|H(t)| \leq C t,
$$

where $C=2 \alpha(\exp (R)-1)(1-\delta)^{-2}$ and $R=\delta+\epsilon_{Q}$. 
Proof. For $0<s<t$ and $t-s \leq 1$, consider $\mathbb{E}[X(s)\{X(t)-X(s)\}]$. For an individual, aged $q$, the infinitesimal rates at which they give birth and die are $\delta$ and $f_{Q}(q)$, respectively. Therefore, a straightforward coupling shows that $\{|X(t)-X(s)|\} \mid X(s)=k \leq_{\text {st }}(W(t)-$ $W(s)) \mid W(s)=k$, where $W(\cdot)$ is a pure birth process in which each individual gives birth at the points of a homogeneous Poisson point process with rate $R$ and ' $\leq_{\mathrm{st}}$ ' denotes stochastically less than. Thus, $(W(t)-W(s))$ is an upper bound for the total number of births and deaths in the branching process in the interval $(s, t]$ with

$$
\begin{aligned}
\mathbb{E}[\{|X(t)-X(s)|\} \mid X(s)=k] & \leq \mathbb{E}[(W(t)-W(s)) \mid W(s)=k] \\
& =k\{\exp (R(t-s))-1\} .
\end{aligned}
$$

Hence,

$$
\begin{aligned}
|\mathbb{E}[X(s)\{X(t)-X(s)\}]| & \leq \mathbb{E}\left[X(s)^{2}\{\exp (R(t-s))-1\}\right] \\
& \leq(t-s)(\exp (R)-1) \mathbb{E}\left[X(s)^{2}\right],
\end{aligned}
$$

since, for $u \leq 1, \exp (R u)-1 \leq u(\exp (R)-1)$. Therefore, for $0 \leq t \leq 1$,

$$
\begin{aligned}
|H(t)| & =\left|2 \alpha \int_{-\infty}^{t} \mathbb{E}[X(-u)(X(-u)-X(t-u))] \mathrm{d} u\right| \\
& \leq 2 \alpha \int_{-\infty}^{t}|\mathbb{E}[X(-u)(X(-u)-X(t-u))]| \mathrm{d} u \\
& \leq 2 \alpha \int_{-\infty}^{t} t(\exp (R)-1) \mathbb{E}\left[X(-u)^{2}\right] \mathrm{d} u \\
& \leq 2 \alpha t(\exp (R)-1) \int_{0}^{\infty} \mathbb{E}\left[X(u)^{2}\right] \mathrm{d} u \\
& \leq 2 \alpha t \frac{\exp (R)-1}{(1-\delta)^{2}} \\
& =C t
\end{aligned}
$$

completing the proof.

The condition $\epsilon_{Q}<\infty$ is almost certainly not required for (2.13) to hold. For $Q \equiv 1$, and any small $\varepsilon>0, \mathbb{E}[|X(1+\varepsilon)-X(1-\varepsilon)| \mid X(1-\varepsilon)] \approx 1$, which prevents a bound of the form (2.14). Dealing with this technicality should not prove difficult but is cumbersome taking into account when individuals are born as well as the number alive at time $t$ and, hence, we omit a proof.

Theorem 2.1. We have

$$
Z_{N} \Rightarrow Z \text { on } D[0, \infty) \text { as } N \rightarrow \infty
$$

Proof. Fix $T \geq 0$. By Lemma 2.4 and Corollary 25.6 of Rogers and Williams (1994, Chapter I), $Z$ has almost surely continuous sample paths.

By Chebychev's inequality, for any $\varepsilon_{N} \rightarrow 0$ as $N \rightarrow \infty, 0 \leq t \leq T$, and $\epsilon>0$,

$$
\begin{aligned}
\mathbb{P}\left(\left|Z_{N}\left(t+\varepsilon_{N}\right)-Z_{N}(t)\right|>\epsilon\right) & =\mathbb{P}\left(\left|Z_{N}\left(\varepsilon_{N}\right)-Z_{N}(0)\right|>\epsilon\right) \\
& \leq \frac{1}{\epsilon^{2}} H\left(\varepsilon_{N}\right) \\
& \rightarrow 0 \text { as } N \rightarrow \infty
\end{aligned}
$$


Thus, Condition (A) of Aldous (1978) holds and, since $Z$ has almost surely continuous sample paths and the finite-dimensional distributions of $\left\{Z_{N}\right\}$ converge to those of $Z$, it follows from Corollary 1 of Aldous (1978) that

$$
Z_{N} \Rightarrow Z \quad \text { on } D[0, T] \text { as } N \rightarrow \infty .
$$

Given that (2.15) holds for all $T>0$, the theorem follows by Theorem $3^{\prime}$ of Lindvall (1973).

\section{SIS epidemic}

We turn our attention to the SIS epidemic model. For the Markov SIS epidemic model, it is known that, as $N \rightarrow \infty$, the proportion infected in the endemic equilibrium is $\rho=1-1 / R_{0}$, where $R_{0}=\beta \mathbb{E}[Q](=\beta)>1$. (For $R_{0} \leq 1$, there is no endemic equilibrium.) Hence, for $\mathbb{E}[Q]=1$, the endemic equilibrium is $\rho=(\beta-1) / \beta$, and the mean of the quasistationary distribution is approximately $N \rho$. Furthermore, the quasistationary distribution can be obtained in this case; see Equation (4) of Clancy and Pollett (2003). However, the quasistationary distribution is cumbersome, hence, the search for simple but good approximations (see Kryscio and Lefèvre (1989), Nåsell (1996), (1999a), Clancy and Pollett (2003), Clancy and Mendy (2011), and Clancy (2012)). In Clancy and Mendy (2011) a range of approximations are considered for the supercritical case $R_{0}>1$, including a negative binomial approximation in the range $1<R_{0}<2\left(0<\rho<\frac{1}{2}\right)$, which is the most common practical situation. A negative binomial approximation based upon the distribution of $B_{N}^{*}$ derived in Section 2 for an appropriate choice of $\alpha$ and $\delta$ will be used here and differs from the negative binomial approximation obtained in Clancy and Mendy (2011). We provide a correction $\tilde{Y}_{N}$ to $B_{N}^{*}$ which takes into account the overestimation of the number infected in the quasistationary distribution using $B_{N}^{*}$. In particular, $\mathbb{E}\left[\tilde{Y}_{N}\right]$ agrees with the means of the approximating quasistationary distributions derived in Clancy and Mendy (2011, Section 3.2) up to but not including a term of $O(1 / N)$. First, we consider $Z_{N}^{*}$ and $Z_{N}(\cdot)$ and their limits as $N \rightarrow \infty$ for normalised versions of the total number of infectives in Theorem 3.1. For large $N, Z_{N}^{*}$ and $Z_{N}(\cdot)$ are shown to provide very good approximations to the epidemic process.

For $N \geq 1$, the epidemic $\varepsilon_{N}$ is the homogeneously mixing epidemic in a population size $N$, where individuals have i.i.d. infectious periods according to $Q$ and infectious indiviiduals make infectious contacts at the points of a homogeneous Poisson point process with rate $\beta$. The individual contacted by an infectious contact is equally likely to be any member of the population, including the infective making the contact. An infectious contact with a susceptible results in the individual becoming infected and instantaneously infectious, whilst infectious contacts with infectives have no affect on the recipient. At the end of their infectious period an infective returns to the susceptible state and consequently individuals can be infected multiple times.

Let $Y_{N}(t)$ be the total number of infectives in $\varepsilon_{N}$ at time $t$, and let $W_{N}(t)=\sqrt{N}\left(Y_{N}(t) / N-\right.$ $\rho)$. Then $Y_{N}(t)=N \rho+\sqrt{N} W_{N}(t)$ and the infinitesimal infection rate at time $t$ is

$$
\begin{aligned}
\frac{\beta}{N} Y_{N}(t)\left(N-Y_{N}(t)\right) & =\frac{\beta}{N}\left(N \rho+\sqrt{N} W_{N}(t)\right)\left(N-N \rho-\sqrt{N} W_{N}(t)\right) \\
& =\frac{\beta}{N} N^{2} \rho(1-\rho)+\frac{\beta}{N} N^{3 / 2} W_{N}(t)(1-2 \rho)-\beta W_{N}(t)^{2} \\
& =\frac{\beta}{N} N^{2} \rho(1-\rho)+\frac{\beta}{N} N\left(Y_{N}(t)-N \rho\right)(1-2 \rho)-\beta W_{N}(t)^{2} \\
& =N \beta \rho^{2}+(2-\beta) Y_{N}(t)-\beta W_{N}(t)^{2} .
\end{aligned}
$$


Given (3.1), for $1<\beta\left(=R_{0}\right)<2$, we couple $\mathcal{E}_{N}$ to $\mathcal{B}_{N}$ with immigration rate $\alpha N=\beta \rho^{2} N$ and birth rate $\delta=2-\beta<1$. Suppose that $Y_{N}(0) \approx \rho N$ and that $W_{N}(t)$ is similar to $Z_{N}(t)$ in having a Gaussian limit as $N \rightarrow \infty$. Then the first two terms on the right-hand side of (3.1) are $O(N)$ and the last term is $O(1)$ with high probability. Therefore, $N \beta \rho^{2}+(2-\beta) Y_{N}(t)$ gives a linear approximation and upper bound, since $\beta W_{N}(t)^{2} \geq 0$, to the infection rate $\beta Y_{N}(t)\left(N-Y_{N}(t)\right) / N$ which we will exploit. If $Y_{N}(t) \leq B_{N}(t)$, the birth rate at time $t$ in $\mathscr{B}_{N}$ is $N \beta \rho^{2}+(2-\beta) B_{N}(t)$ and a simple coupling can be made between $\mathscr{B}_{N}$ and $\mathcal{E}_{N}$ such that a birth at time $t$ in $\mathscr{B}_{N}$ has a corresponding infection in $\mathcal{E}_{N}$ with probability

$$
\frac{N \beta \rho^{2}+(2-\beta) Y_{N}(t)-\beta W_{N}(t)^{2}}{N \beta \rho^{2}+(2-\beta) B_{N}(t)}=1-\left\{O\left(\frac{W_{N}(t)^{2}}{N}\right)+O\left(\frac{B_{N}(t)-Y_{N}(t)}{N}\right)\right\} .
$$

This is the key to using the subcritical branching process with immigration approximation for the epidemic process. Thus, we compare $Y_{N}(t)$ and $W_{N}(t)$ with $B_{N}(t)$ and $Z_{N}(t)$, respectively.

For any $N, Y_{N}(t) \rightarrow 0$ a.s. as $t \rightarrow \infty$ and, therefore, we are interested in the quasistationary distribution of $Y_{N}, Y_{N}^{*} \stackrel{\mathrm{D}}{=}\left\{Y_{N}(t) \mid Y_{N}(t)>0\right\}_{t \rightarrow \infty}$. A natural approximation of $Y_{N}^{*}$, regardless of the choice of $Q$, is $B_{N}^{*} \sim \operatorname{NBin}\left(N \beta \rho^{2} /(2-\beta), \beta-1\right)$ which gives $\mathbb{E}\left[Y_{N}^{*}\right] \approx N \rho$ and $\operatorname{var}\left(Y_{N}^{*}\right) \approx N / \beta$. Since $\mathcal{E}_{N}$ and $\mathscr{B}_{N}$ can easily be coupled such that the lifetimes (infectious periods) of individuals are identical in both processes at time 0 and, for all $t>0, Y_{N}(t) \leq B_{N}(t)$, it is clear that $\mathbb{E}\left[Y_{N}^{*}\right] \leq N \rho$. We return later to give an improved approximation $\tilde{Y}_{N}$ of $Y_{N}^{*}$ and, hence, $\mathbb{E}\left[Y_{N}^{*}\right]$ taking into account the last term in (3.1).

For the epidemic $\mathcal{E}_{N}$, we initialise the population at time 0 by drawing the number of infectives and the remaining lengths of the infectious periods of the infectives from the stationary distribution of $\mathscr{B}_{N}$. For large $N$, the probability of attempting to start with 0 or more than $N$ infectives is negligible. Thus, $Y_{N}(0)=B_{N}(0)$ and $W_{N}(0)=Z_{N}(0)$, and whenever an initial individual (alive at time 0 ) dies in $\mathscr{B}_{N}$, the corresponding individual recovers in $\mathcal{E}_{N}$. By exploiting the coupling given above using (3.2), and following Section 2 and Scalia-Tomba (1990) by defining the processes $W_{N}$ and $Z_{N}$ for $N \geq 1$, on $D[0, \infty)$ endowed with the Skorokhod topology we proceed by proving Theorem 3.1.

Theorem 3.1. We have

$$
W_{N} \Rightarrow Z \text { on } D[0, \infty) \text { as } N \rightarrow \infty
$$

where $Z$ denotes the Gaussian limit of $Z_{N}$ obtained in Section 2 with covariance function given by (2.6).

To prove Theorem 3.1, we introduce a difference process $\mathcal{D}_{N}$ with $D_{N}(t)=B_{N}(t)-Y_{N}(t)$, the difference between the total number of individuals alive in $\mathscr{B}_{N}$ and the total number of individuals infectious in $\mathcal{E}_{N}$ at time $t$. Individuals arrive into $\mathscr{D}_{N}$ at the points of an inhomogeneous Poisson point process with rate

$$
(2-\beta) D_{N}(s)+\beta W_{N}(s)^{2}=(2-\beta) D_{N}(s)+\beta\left(-D_{N}(s)+Z_{N}(s)\right)^{2} .
$$

The lifetimes of individuals arriving in $\mathscr{D}_{N}$ are distributed according to $Q$.

Now, for $t \geq 0, Z_{N}(t)-W_{N}(t)=D_{N}(t) / \sqrt{N}$. Then, for any $T>0$,

$$
W_{N} \Rightarrow Z \quad \text { on } D[0, T] \text { as } n \rightarrow \infty,
$$

follows from Theorem 2.1 by Theorem 4.1 of Billingsley (1968) if

$$
\sup _{0 \leq t \leq T}\left|\frac{D_{N}(t)}{\sqrt{N}}\right| \rightarrow 0 \quad \text { as } N \rightarrow \infty .
$$


To prove (3.3), it is useful to bound $D_{N}(t)$ from above by the total number of individuals, $U_{A}(t)$, in a pure birth-immigration process. Let $U_{A}(0)=0$, and suppose that individuals arrive in the birth-immigration process at rate $U_{A}(t)+A$. It is trivial to show that $\mathbb{E}\left[U_{A}(t)\right]=$ $A\{\exp (t)-1\}$ and, hence, by Markov's inequality, for any $A>0$ and all $\epsilon>0$,

$$
\begin{aligned}
\mathbb{P}\left(\sup _{0 \leq t \leq T} U_{A}(t)>\epsilon \sqrt{N}\right) & =\mathbb{P}\left(U_{A}(T)>\epsilon \sqrt{N}\right) \\
& \leq \frac{1}{\epsilon \sqrt{N}} \mathbb{E}\left[U_{A}(T)\right] \\
& \rightarrow 0 \quad \text { as } N \rightarrow \infty
\end{aligned}
$$

Lemma 3.1. For any $T>0$,

$$
\sup _{0 \leq t \leq T}\left|\frac{D_{N}(t)}{\sqrt{N}}\right| \rightarrow 0 \quad \text { as } N \rightarrow \infty .
$$

Proof. Fix $T, \epsilon, \delta>0$, and choose $A_{\delta}>0$ such that, for all sufficiently large $N$,

$$
\mathbb{P}\left(\sup _{0 \leq t \leq T} Z_{N}(t)^{2}>A_{\delta}\right) \leq \frac{\delta}{2}
$$

This can be done since $Z_{N} \Rightarrow Z$ on $D[0, T]$ as $N \rightarrow \infty$ by Theorem 2.1.

Using the Cauchy-Schwarz inequality and conditional upon $\sup _{0 \leq t \leq T} Z_{N}(t)^{2} \leq A_{\delta}$, it follows that, for $0 \leq t \leq T$, the birth rate in $\mathscr{D}_{N}$ on the interval $[0, T]$ is bounded above by

$$
(2-\beta) D_{N}(s)+\frac{2 \beta}{N} D_{N}(s)^{2}+2 \beta A_{\delta} .
$$

Also while $(2 \beta / N) D_{N}(s) \leq \beta-1$, the birth rate in $\mathcal{D}_{N}$ is bounded above by $D_{N}(s)+2 \beta A_{\delta}$.

Therefore, if we let $A=2 \beta A_{\delta}, \sup _{0 \leq t \leq T} Z_{N}(t)^{2} \leq A_{\delta}$ and $U_{A}(T) \leq N(\beta-1) /(2 \beta)$ together imply that $\sup _{0 \leq t \leq T} D_{N}(t) \leq U_{A}(\bar{T})$.

For all sufficiently large $N, \epsilon \sqrt{N} \leq N(\beta-1) /(2 \beta)$ and, hence,

$$
\begin{aligned}
\mathbb{P}\left(\sup _{0 \leq t \leq T}\left|\frac{D_{N}(t)}{\sqrt{N}}\right|>\epsilon\right) \\
\quad \leq \mathbb{P}\left(U_{A}(T)>\epsilon \sqrt{N}, \sup _{0 \leq t \leq T} Z_{N}(t)^{2} \leq A_{\delta}\right)+\mathbb{P}\left(\sup _{0 \leq t \leq T} Z_{N}(t)^{2}>A_{\delta}\right) \\
\quad \leq \mathbb{P}\left(U_{A}(T)>\epsilon \sqrt{N}\right)+\mathbb{P}\left(\sup _{0 \leq t \leq T} Z_{N}(t)^{2}>A_{\delta}\right),
\end{aligned}
$$

with the right-hand side of (3.6) being less than $\delta$ for all sufficiently large $N$ by (3.5) and (3.4).

Proof of Theorem 3.1. Fix $T>0$. By Theorem 2.1, Lemma 3.1, and Billingsley (1968, Theorem 4.1),

$$
W_{N} \Rightarrow Z \quad \text { on } D[0, T] \text { as } N \rightarrow \infty .
$$

Since (3.7) holds for all $T>0$, the theorem follows from Lindvall (1973, Theorem $3^{\prime}$ ).

A corollary of Theorem 3.1 is that

$$
W_{N}^{*}=\sqrt{N}\left(\frac{Y_{N}^{*}}{N}-\rho\right) \stackrel{\mathrm{D}}{\rightarrow} Z^{*} \sim N\left(0, \frac{1}{\beta}\right) \quad \text { as } N \rightarrow \infty .
$$


Returning to $\mathbb{E}\left[Y_{N}^{*}\right]$ for finite $N$, we use Theorem 3.1 to give an improved approximation. Note that $Y_{N}^{*}=B_{N}^{*}-D_{N}^{*}$ with $\mathbb{E}\left[B_{N}^{*}\right]=N \rho$ and $D_{N}^{*} \stackrel{\mathrm{D}}{=}\left\{D_{N}(t) \mid D_{N}(t)>0\right\}_{t \rightarrow \infty}$, the quasistationary distribution of the difference process. Now $\mathscr{D}_{N}$ evolves with birth rate $(2-$ $\beta) D_{N}(t)+\beta W_{N}(t)^{2}$, that is, a subcritical branching process with a time inhomogeneous immigration rate. For $s \geq 0$, let $G_{Q}(s)=\mathbb{P}(Q>s)$, the probability the infectious period of an individual exceeds $s$ units of time. Hence,

$$
\begin{aligned}
\mathbb{E}\left[D_{N}(t)\right] & =\int_{0}^{t} \mathbb{E}\left[(2-\beta) D_{N}(s)+\beta W_{N}(s)^{2}\right] G_{Q}(t-s) \mathrm{d} s \\
& =\int_{0}^{t}(2-\beta) \mathbb{E}\left[D_{N}(s)\right] G_{Q}(t-s) \mathrm{d} s+\int_{0}^{t} \beta \mathbb{E}\left[W_{N}(s)^{2}\right] G_{Q}(t-s) \mathrm{d} s
\end{aligned}
$$

Whilst the distribution of $D_{N}(t)$ depends upon $t$, for large $t,\left\{D_{N}(t) \mid D_{N}(t)>0\right\} \stackrel{\mathrm{D}}{\approx} D_{N}^{*}$. Also, using Theorem 3.1, we can replace $W_{N}(s)$ by $Z(s) \stackrel{\text { D }}{=} Z^{*}$, giving, for large $t$, with $u=t-s$,

$$
\begin{aligned}
\mathbb{E}\left[D_{N}(t)\right] & \approx \mathbb{E}\left[D_{N}^{*}\right] \\
& \approx \int_{0}^{\infty}(2-\beta) \mathbb{E}\left[D_{N}^{*}\right] G_{Q}(u) \mathrm{d} u+\int_{0}^{\infty} \beta \mathbb{E}\left[\left(Z^{*}\right)^{2}\right] G_{Q}(u) \mathrm{d} u \\
& =(2-\beta) \mathbb{E}\left[D_{N}^{*}\right] \int_{0}^{\infty} G_{Q}(u) \mathrm{d} u+\beta \mathbb{E}\left[\left(Z^{*}\right)^{2}\right] \int_{0}^{\infty} G_{Q}(u) \mathrm{d} u,
\end{aligned}
$$

where $\int_{0}^{\infty} G_{Q}(u) \mathrm{d} u=\mathbb{E}[Q]=1$ and $\mathbb{E}\left[\left(Z^{*}\right)^{2}\right]=1 / \beta$. Hence, $\mathbb{E}\left[D_{N}^{*}\right] \approx(2-\beta) \mathbb{E}\left[D_{N}^{*}\right]+$ $\beta / \beta$, giving $\mathbb{E}\left[D_{N}^{*}\right] \approx 1 /(\beta-1)$. Therefore, $\mathbb{E}\left[Y_{N}^{*}\right] \approx N \rho-1 /(\beta-1)$ which agrees with Clancy and Mendy (2011, Section 3.2) and Nåsell (2003) up to an $O(1 / N)$ term. Similarly, $\operatorname{var}\left(Y_{N}^{*}\right)=N / \beta$ agrees with that given in Clancy and Mendy (2011, Section 3.2) up to an $O(1)$ term. The key difference being that the construction of our approximation is not restricted to $Q \sim \operatorname{Exp}(1)$.

Finally, the above motivates approximating $Y_{N}^{*}$ by $\tilde{Y}_{N} \sim \operatorname{NBin}\left(N \beta \rho^{2} /(2-\beta)-1, \beta-1\right)$ to take account of the overestimation of $Y_{N}^{*}$ by $B_{N}^{*}$ with $\mathbb{E}\left[\tilde{Y}_{N}\right]=N \rho-1 /(\beta-1)$. The exact quasistationary distribution is difficult to obtain for nonexponential infectious periods. However, simulations can be used to estimate $Y_{N}^{*}$. Simulations for $N$ between 100 and 500 with $N \rho>50$ showed that $d_{\mathrm{TV}}\left(Y_{N}^{*}, \tilde{Y}_{N}\right)$ was typically about two-thirds of $d_{\mathrm{TV}}\left(Y_{N}^{*}, \tilde{B}_{N}\right)$, where $d_{\mathrm{TV}}(\cdot, \cdot)$ denotes total variation distance. This was confirmed for exponentially distributed infectious periods, where comparisons can be made with the approximations of the quasistationary distribution obtained in Clancy and Mendy (2011). In Figure 1, the approximations $\tilde{Y}_{N}$ and $B_{N}^{*}$ are compared with the negative binomial and beta-binomial approximations proposed in Clancy and Mendy (2011) over $1<\beta<2$ for $N=50$. Note that, as $\beta \rightarrow 2$, both $\tilde{Y}_{N}$ and $B_{N}^{*}$ converge to $\operatorname{Po}(N / 2)$. We observe that only the approximation $B_{N}^{*}$ is defined for $\beta \leq 1.14$ and this approximation is best for $\beta \leq 1.3$. For $\beta$ around 1.4, the approximation $\tilde{Y}_{N}$ is best and, for $\beta>1.46$, the beta-binomial approximation of Clancy and Mendy (2011) performs best. More generally, for $N \rho>25$, the beta-binomial approximation of Clancy and Mendy (2011) is preferable, although the negative binomial approximations given in this paper consistently outperform the negative binomial approximation of Clancy and Mendy (2011) especially for $N \rho<25$ when such an approximation is particularly useful. The distribution of $B_{N}^{*}$ is independent of $Q$, which supports using the approximating quasistationary distributions obtained in Clancy and Mendy (2011) and elsewhere for general $Q$. 


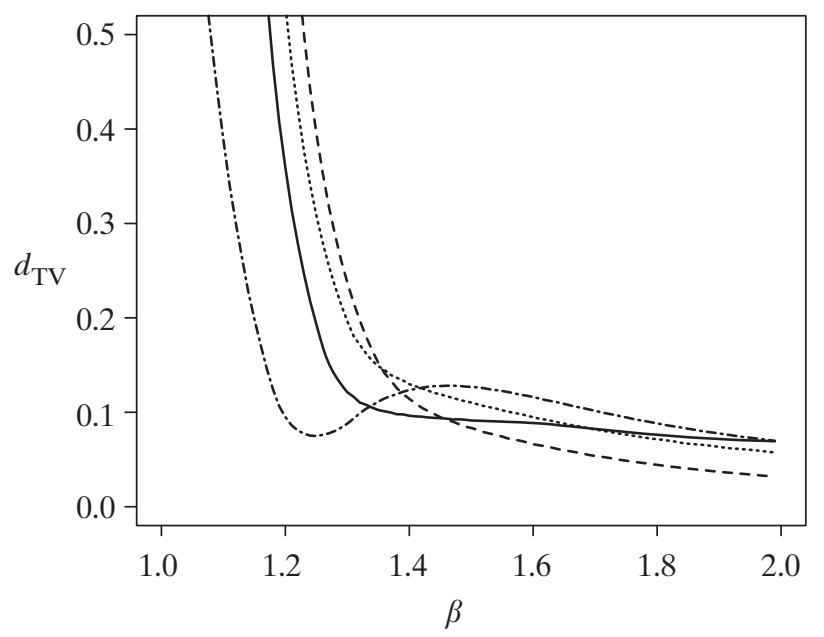

FIGURE 1: Plot of $d_{\mathrm{TV}}$ against $\beta$ for $\tilde{Y}_{N}$ (solid line), $B_{N}^{*}$ (dash-dot line), negative binomial Clancy and Mendy (2011) (dotted line), and beta-binomial Clancy and Mendy (2011) (dashed line) with $N=50$.

\section{Conclusions}

Throughout this paper we have sought to give insight to the endemic behaviour of an SIS epidemic with a general infectious period distribution. As noted in Section 1, the branching process approximation for the early stages of an SIR epidemic continues to hold for an SIS epidemic and it is therefore straightforward to compute key quantities such as $R_{0}$ and the probability of (early) extinction of the epidemic in terms of the infectious period distribution. The branching process approximation is based upon a linearisation of the epidemic process and it is the same idea that is employed in this paper, not to the initial stages of the epidemic but to the endemic level. This allowed us to derive a simple approximation in Section 2 and to obtain interesting results for the resulting subcritical branching process with immigration. In particular, that the distribution of $B_{N}^{*}$ depends only upon $\alpha, \delta$, and $\mathbb{E}[Q]$ and not the distributional form of $Q$.

The evolution of $B_{N}(t)$ and, consequently, $Z_{N}(t)$ do depend upon $Q$. This suggests that the time to extinction from the endemic equilibrium will depend upon $Q$. A small simulation study with $N=200$ and $\beta=1.25$ was conducted to observe the effect of the distribution of $Q$ on the mean time to extinction. It was observed that the mean time to extinction increased as $\operatorname{var}(Q)$ increased. This is worthy of further consideration, but quantitative results concerning the time to extinction are limited; see Andersson and Djehiche (1998) for an exception. In Nåsell (1999a) and Andersson and Britton (2000) for SIR epidemics with demography and in Britton and Neal (2010) for SIS household epidemics, the coefficient of variation of the Gaussian endemic limit has been used to give a qualitative assessment of the time to extinction for different sets of parameters. This approach is not appropriate here as, for a given $\beta$, all choices of $Q$ with a given $\mathbb{E}[Q]$ will have the same Gaussian endemic limit and, hence, coefficient of variation. For a better understanding of the effect of $Q$ on the time to extinction, it is necessary to study the behaviour of $v(s, t)=\mathbb{E}[X(s) X(t)]$ and its impact on the covariance of $Z(t)$ given by (2.6). In particular, using the Gaussian approximation for studying the time to extinction is equivalent to studying the first passage of a (stationary) Gaussian process across some extremal value. For a fixed $\beta$ and, hence, a fixed mean and variance of $Z(t)$, the first passage time will generally take 
longer if $\operatorname{cov}(Z(0), Z(t))$ is larger, and it is hence conjectured that the mean extinction time will be increasing as $\operatorname{cov}(Z(0), Z(t))$ increases. However, given the form of $\operatorname{cov}(Z(0), Z(t))$ in (2.6), it is difficult to make simple, general statements about the effect of the distributional form of $Q$ on $\operatorname{cov}(Z(0), Z(t))$.

\section{Acknowledgement}

This research was supported in part by the UK Engineering and Physical Sciences Research Council (EPSRC), under research grant EP/J008443/1.

\section{References}

Aldous, D. (1978). Stopping times and tightness. Ann. Prob. 6, 335-340.

ANDERSSON, H. AND BRITTON, T. (2000). Stochastic epidemics in dynamic populations: quasi-stationarity and extinction. J. Math. Biol. 41, 559-580.

Andersson, H. And Djehiche, B. (1998). A threshold limit theorem for the stochastic logistic epidemic. J. Appl. Prob. 35, 662-670.

Arrigoni, F. ANd Pugliese, A. (2007). Global stability of equilibria for a metapopulation S-I-S model. In Math Everywhere, Springer, Berlin, pp. 229-240.

BALL, F. (1999). Stochastic and deterministic models for SIS epidemics among a population partitioned into households. Math. Biosci. 156, 41-67.

Ball, F. And Donnelly, P. (1995). Strong approximations for epidemic models. Stoch. Process. Appl. 55, 1-21.

Billingsley, P. (1968). Convergence of Probability Measures. John Wiley, New York.

Britton, T. and Neal, P. (2010). The time to extinction for a stochastic SIS-household-epidemic model. J. Math. Biol. 61, 763-779.

Clancy, D. (2012). Approximating quasistationary distributions of birth-death processes. J. Appl. Prob. 49, 10361051.

Clancy, D. And Mendy, S. T. (2011). Approximating the quasistationary distribution of the SIS model for endemic infection. Methodol. Comput. Appl. Prob. 13, 603-618.

Clancy, D. And Pollett, P. K. (2003). A note on quasistationary distributions of birth-death processes and the SIS logistic epidemic. J. Appl. Prob. 40, 821-825.

Ghoshal, G., SANDER, L. M. ANd SoKolov, I. M. (2004). SIS epidemics with household structure: the self-consistent field method. Math. Biosci. 190, 71-85.

Kryscio, R. And Lefèvre, C. (1989). On the extinction of the S-I-S stochastic logistic epidemic. J. Appl. Prob. 26, 685-694.

KurTz, T. G. (1971). Limit theorems for sequences of jump Markov processes approximating ordinary differential processes. J. Appl. Prob. 8, 344-356.

LiNDVALL, T. (1973). Weak convergence of probability measures and random functions in function space on $D(0, \infty)$. J. Appl. Prob. 10, 109-121.

NÅSEll, I. (1996). The quasistationary distribution of the closed endemic SIS model. Adv. Appl. Prob. 28, 895-932.

NÅSELL, I. (1999a). On the quasistationary distribution of the stochastic logistic epidemic. Math. Biosci. 156, 21-40.

NÅSELl, I. (1999b). On the time to extinction in recurrent epidemics. J. R. Statist. Soc. B 61, 309-330.

NÅsell, I. (2003). An extension of the moment closure method. Theoret. Pop. Biol. 64, 233-239.

Neal, P. (2006). Stochastic and deterministic analysis of SIS household epidemics. Adv. Appl. Prob. 38, 943-968. (Correction: 44 (2012), 309-310.)

Neal P. (2008). The SIS great circle epidemic model. J. Appl. Prob. 45, 513-530.

Rogers, L. C. G. AND Williams, D. (1994). Diffusions, Markov Processes, and Martingales, Vol. 1, Foundations. 2nd edn. John Wiley, Chichester.

Scalia-Tomba, G. (1990). On the asymptotic final size distribution of epidemics in heterogeneous populations. In Stochastic Processes in Epidemic Theory, Springer, New York., pp. 189-196.

Weiss, G. H. AND Dishon, M. (1971). On the asymptotic behavior of the stochastic and deterministic models of an epidemic. Math. Biosci. 11, 261-265. 